\title{
Análise e Simulação de Ondas Sonoras Assistidas por Computador
}

(Computer-aided sound wave analysis and simulation)

\author{
Lucas Bleicher \\ Departamento de Física, Universidade Federal do Ceará \\ Rua Oswaldo Cruz, 635/701, Meireles, CEP 60125-150 Fortaleza-Ce \\ lbleicher@yahoo.com \\ Moésio Medeiros da Silva, Júlio Wilson Ribeiro \\ Departamento de Computação, Universidade Federal do Ceará \\ moesio@padetec.ufc.br; jwilson@lia.ufc.br \\ Márcio Gurjão Mesquita \\ Departamento de Estatística e Computação, Universidade Estadual do Ceará \\ marciomesquita@yahoo.com
}

Recebido em 7 de março, 2002. Aceito em 26 de maio, 2002.

\begin{abstract}
Como ferramenta auxiliar no ensino de Física, é apresentada a utilização do software de computação simbólica Mathematica, através dos seus recursos de programação analítica e multimídia. É possível verificar as relações de freqüências numa escala musical e o efeito do batimento, através de análise matemática e reprodução sonora via computador. Para fins ilustrativos e pedagógicos, são simulados no computador efeitos sonoros comumente utilizados por músicos, como Tremolo, Phaser e AutoWah. Estes são modelados matematicamente de forma simplificada, tomando-se como base uma onda sonora padrão, cujos parâmetros associados foram obtidos experimentalmente. Ressalta-se a possibilidade do aprendizado autônomo.
\end{abstract}

As an auxiliary tool in Physics teaching, the utilization of analytical programming and multimedia facilities of the symbolic computing software Mathematica is shown. It is possible to verify the frequency ratios in a musical scale and the beat effect by mathematical analysis and audible reproduction by the computer. For illustrative and pedagogical purposes, sound effects commonly used by musicians, such as Tremolo, Phaser and Auto-Wah are simulated. A simplified mathematical model is defined for them using a standard sound wave whose parameters were experimentally obtained. The possibility of self-teaching is evident.

\section{A música e a ciência}

O estudo da relação entre a música e as ciências físicas vem desde a antigüidade. Consta que Pitágoras [1] dedicou muito de seu tempo ao estudo dos intervalos musicais em um instrumento chamado monocórdio, que consiste apenas em uma corda sonora, presa em suas duas extremidades, e um dispositivo móvel, que permite que se faça vibrar apenas uma fração da corda. Um esquema de um monocórdio pode ser visto na Fig. 1.

Pitágoras, experimentalmente, observou que dividindo-se a corda exatamente ao meio (para tanto se fixa o dispositivo móvel na metade do comprimento da corda) e tocando-se a mesma, escutava-se o intervalo da oitava em relação à nota original. Outros intervalos importantes, como a quinta justa e a quarta justa, eram obtidos também por frações de números pequenos (relações de $3 / 2$ e $4 / 3$ respectivamente). Os pitagóricos, então, desenvolveram um método de gerar novas notas a partir das conhecidas: dividindo ou multiplicando uma dessas relações por $3 / 2$, e tomando sua metade, se o resultado for maior que dois, ou dobrá-lo, no caso dela ser menor que um. Com isso pode-se obter a escala diatônica, base de praticamente toda a música ocidental. 


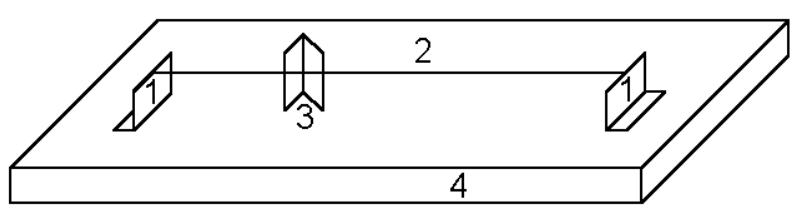

1 - Extremidades de Fixação

2 - Corda sonora

3 - Dispositivo Móvel

4 - Base de Apoio

Figura 1. Visualização esquemática de um monocórdio.

O tratado mais importante sobre a relação entre a Música e a Física foi escrito no século XIX, por Hermann Helmholtz [2]. Tendo estudado ele também Medicina, seu livro Die Lehre von den Tonempfindungen não apenas trata da natureza física da consonância e dissonância dos sons, mas também da forma como o ouvido humano analisa essas particularidades.

No século seguinte, o desenvolvimento da Física tornou-se crucial para a construção de novos instrumentos musicais, como a guitarra elétrica e os sintetizadores. Além disso, o advento da computação tornou possível a pesquisa em algoritmos com as mais diversas aplicações para a música, citando-se a emulação da criatividade em improvisação através da inteligência artificial [3] e a análise de espectros sonoros por funções iterativas [4].

\section{O software Mathematica e a simulação de ondas sonoras}

A manipulação simbólica de dados constitui um recurso primordial do software Mathematica, o qual também inclui diversos tipos de expressões analíticas, possibilitando resultados exatos, o que necessariamente não é possível quando se utilizam métodos puramente numéricos. Além disso, códigos simbólicos assim implementados apresentam uma diversa gama de aplicações, inclusive recursos de multimídia, permitindo, por exemplo, visualizar a variação de um campo ao longo do tempo e/ou mostrar, através de saídas de caixas de som do computador, o comportamento de uma função de onda sonora definida matematicamente pelo usuário, o que será mostrado neste trabalho.

No estudo de ondulatória, o conceito de Onda Senoidal é apresentado como primeiro exemplo de onda. A fórmula geral de uma onda senoidal [5] é representada pela função mostrada a seguir.

$$
y(t)=A \cdot \operatorname{sen}(2 \pi f t+\phi)
$$

Onde $y$ expressa a amplitude, $A$ a amplitude máxima, $f$ a freqüência e $\phi$ a fase.
A função Play, disponível no Mathematica, disponibiliza para o usuário, na forma de som audível, qualquer função matemática, programada pelo mesmo. Programando-se o comando abaixo, é possível ouvir a nota $L a ́$ :

\section{Play $[\operatorname{Sin}[440 * 2 * \pi * t],\{\mathbf{t}, 0,1\}]$}

Na linha de programação acima, a freqüência da nota simulada é de $440 \mathrm{~Hz}$, enquanto o segundo termo do comando, $\{t, 0,1\}$, indica que t é a variável que corresponde ao tempo físico, e que o intervalo de simulação adotado para fins experimentais é de 0 a 1 segundo. Variando-se o valor da freqüência é possível verificar, por exemplo, quais os valores audíveis e compará-los com os valores numéricos fornecidos segundo tabelas disponibilizadas na literatura.

\section{O fenômeno do batimento}

O fenômeno do batimento, que ocorre quando duas ondas de freqüências próximas são sobrepostas, pode ser facilmente simulado no Mathematica. Seu resultado é um som percebido pelo ouvido como a média das duas freqüências, apresentando uma variação de amplitude numa freqüência que é a diferença entre as freqüências das duas ondas. Para fins educacionais, no exemplo definido abaixo, o comando sobrepõe duas ondas senoidais de freqüências $278 \mathrm{~Hz}$ e $275 \mathrm{~Hz}$.

\section{Play $[\{\operatorname{Sin}[(278 * 2 * \pi * t)]+\operatorname{Sin}[(275 * 2 * \pi * t)]\},\{\mathbf{t}, 0,5\}]$}

Assim, torna-se possível ouvir claramente a variação de amplitude no som produzido. Se variarmos uma das freqüências para aproximá-la da outra, podemos verificar que a diversidade de amplitude é cada vez mais lenta. Trata-se justamente do procedimento adotado pelos músicos para afinar seus instrumentos: tocando a nota levemente desafinada junto com um padrão, haverá produção de batimentos. Ajustando a afinação do instrumento, os batimentos vão se tornando cada vez mais lentos, até cessarem por completo, quando a nota estiver perfeitamente afinada segundo o padrão de acuidade humana. Desta forma, torna-se possível analisar matematicamente para que valor numérico converge o erro relativo entre as freqüências de batimento quando o ouvido humano já não mais percebe variações de amplitude.

\section{Modelagem de ondas se- gundo classes de efeitos sonoros}

Foi desenvolvido um procedimento científico para consolidar o processo de aprendizagem do aluno, visando permitir o contato concomitante com a conceituação física e musical de ondas e sua respectiva modelagem 
matemática simplificada. Como complemento a este e visando valorizar aspectos artístico-culturais, foram analisados determinados efeitos sonoros, comumente utilizados em estúdios e apresentações musicais.

$\begin{array}{lllc}\begin{array}{c}\text { Freqüencia } \\ \text { (Hz) }\end{array} & \begin{array}{c}\text { Amplitude } \\ \text { (u.a.) }\end{array} & \begin{array}{c}\text { Fase } \\ \text { (graus) }\end{array} & \begin{array}{c}\text { Amplitude } \\ \text { Normalizada }\end{array} \\ 107.4 & 0.02593 & -25.4 & 0.42 \\ 214.8 & 0.05005 & -125 & 0.81 \\ 322.3 & 0.06166 & -220 & 1.00 \\ 429.7 & 0.05142 & -300 & 0.83 \\ 537.1 & 0.03100 & -376 & 0.50 \\ 644.5 & 0.01050 & -457 & 0.17 \\ 751.9 & 0.00605 & -272 & 0.10 \\ 859.4 & 0.01149 & -332 & 0.19 \\ 966.8 & 0.01239 & -702 & 0.20 \\ 1074.2 & 0.00639 & -678 & 0.10\end{array}$

Tabela 1: Configuração de harmônicos da onda tomada como padrão

Objetivando-se um bom resultado nas simulações, inicialmente é necessário escolher uma onda mais rica que uma simples senoidal. Sabe-se que as notas produzidas via instrumentos musicais são geralmente compostas por freqüências fundamentais e seus harmônicos associados. A relação entre os harmônicos constitui um dos fatores que contribuem para a definição do timbre de um instrumento musical. Para fins experimentais, a tabela 1 ilustra uma configuração de harmônicos que foi gerada pela excitação da corda lá de uma guitarra Yamaha Eterna EG-303. Estes dados numéricos foram obtidos através da aplicação de uma transformada rápida de Fourier na onda produzida pela guitarra e simultaneamente registrada por um osciloscópio.

Assim, toma-se como modelo matemático aproximado uma onda composta por 10 freqüências, apresentando a seguinte forma:

$$
y_{0}(t)=\sum_{i=1}^{10} A_{i} \cdot \operatorname{sen}\left(2 \pi f_{i} t+\phi_{i}\right)
$$

Numa etapa seguinte, utilizando-se o software $M a$ thematica, executa-se a seguinte linha de programação, para ouvir o sinal representativo da onda descrita pela Eq. 2, que doravante é assumida como padrão:

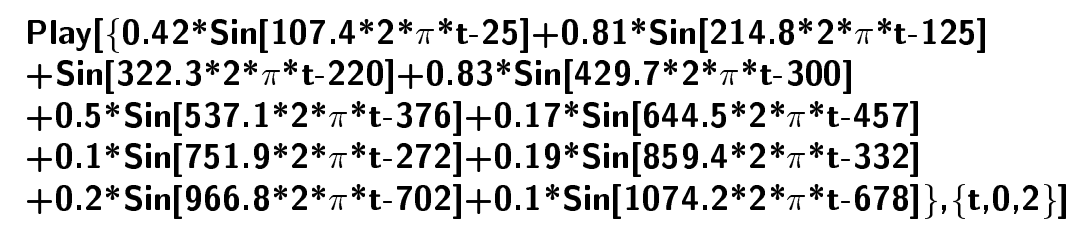

É importante ressaltar que as caixas de som mais comumente usadas em computadores pessoais possuem fraca resposta para freqüências abaixo de $200 \mathrm{~Hz}$. Tais distorções podem ser minimizadas ou mesmo eliminadas utilizando-se fones de ouvido ou caixas de som de melhor qualidade

Para analisar efeitos sonoros comumente utilizados em estúdios e apresentações musicais, são escolhidos três problemas de modelagem, que apresentam crescentes graus de dificuldade em serem computacionalmente modelados e que são descritos a seguir [6].

Tremolo - Esse efeito sonoro produz na onda utilizada como entrada uma variação periódica de amplitude. É como variar periodicamente o botão de volume num aparelho de som. Há dois parâmetros de configuração: a variação máxima de amplitude e a velocidade com que ela varia.

Phaser - O interessante resultado sonoro caracterizado neste efeito é obtido da seguinte forma: soma-se à onda original uma onda de mesma freqüência fe amplitude máxima $A$, mas com uma variação temporal e linear de fase, o que resulta em uma série de inter- ferências construtivas e destrutivas. O único parâmetro desse tipo de efeito é a freqüência com que varia a fase da onda somada à original.

Auto-wah - O nome "wah" tem caráter de onomatopéia para o resultado desse efeito. No "wah" controlado com o pé, a variação que se dá ao pisar no pedal faz com que a onda sonora tenha uma certa região de freqüências acentuada: pisando-se até o fim do pedal, são ouvidas as freqüências mais agudas; voltando-se à posição inicial, ouvem-se as mais graves. No auto-wah, essa variação é feita de forma automática, de maneira que há sempre uma oscilação entre freqüências mais agudas e mais graves. É importante ressaltar que a onda padrão adotada é discretizada (formada apenas por 10 freqüências, ver Eq. 2). Numa analogia semelhante à utilizada no efeito do tremolo, o auto-wah se assemelha a uma variação periódica no botão tone de um aparelho de som. Assim como o tremolo, ele possui dois parâmetros: um que determina a variação máxima da amplitude para as freqüências ressaltadas, e outro que define o quão rápido ocorrerá essa variação.

A modelagem do efeito tremolo é a mais simples das 
três e pode ser escrita da seguinte forma.

$$
y(t)=\left[y_{o}(t)\right] \cdot[1+D \cdot \operatorname{sen}(S . t)](0<D<1)
$$

Onde $y_{o}(t)$ representa a onda padrão, descrita pela Eq. 2. O termo que é multiplicado a $y_{o}(t)$ faz com que a amplitude varie entre $A[1+D]$ e $A[1-D]$, sendo a variação mais rápida ou mais lenta de acordo com o parâmetro $\mathrm{S}^{1}$.

A modelagem seguinte é a do efeito Phaser. Tornase simples modelar o efeito a partir de sua definição: somamos à função $\mathrm{y}_{o}(\mathrm{t})$ uma onda semelhante, porém acrescida de um termo interno à função seno que varie a fase da nova onda. A função é mostrada abaixo:

$$
y(t)=y_{o}(t)+\sum_{i=1}^{10} A_{i} \operatorname{sen}\left(2 \pi \cdot f_{i} \cdot t+S \cdot t\right)
$$

O termo $S$ determina a rapidez com que a fase da segunda onda varia com o tempo.

Finalmente, chegamos à mais complicada dentre as modelagens apresentadas, a do auto-wah. O efeito pode ser modelado de forma análoga ao tremolo. Porém, em vez de variar a amplitude de toda a onda, é preciso amplificar de forma periódica apenas algumas regiões de freqüência. Para solucionar matematicamente este problema, insere-se em cada um dos 10 termos da função $y_{o}(t)$ um fator semelhante ao utilizado na Eq. (3), porém utilizam-se defasagens diferentes para cada termo, o que ressaltará cada uma das dez ondas componentes de forma diferente em cada instante do tempo. A função arbitrada é expressa a seguir:

$$
y(t)=\sum_{i=1}^{10}\left[A_{i} \operatorname{sen}\left(2 \pi f_{i} t+\phi_{i}\right)\right] \cdot\left[1+D \operatorname{sen}\left(R \pi t+\frac{i \pi}{10}\right)\right]
$$

É possível verificar a semelhança entre a Eq. 5, que descreve o auto-wah, e a Eq. 3, que descreve o tremolo. Enquanto na Eq. 3 há um termo $[1+$ D.sen(S.t)] multiplicado a $\mathrm{y}_{o}$, na Eq. 5 esse termo tem adicionalmente uma fase dependente de i e é multiplicado a cada componente do somatório da série que define $\mathrm{y}_{o}$. Isso resulta na amplificação de diferentes freqüências, segundo valores distintos atribuídos ao tempo. Assim como no tremolo, a variação máxima de amplitude é função de $\mathrm{D}$, enquanto $\mathrm{R}$ expressa a velocidade dessa variação.

Finalmente, o resultado sonoro das três novas ondas definidas e representadas pelas Eqs. 3, 4 e 5 pode ser ouvido e analisado com auxílio do software Mathematica, executando-se o comando Play, anteriormente discutido. Para tanto, substituem-se nos parâmetros $A_{i}$, $f_{i}$ e $\phi_{i}$ os valores numéricos obtidos experimentalmente e disponibilizados na tabela 1. Como dados complementares, variam-se convenientemente os parâmetros $\mathrm{S}, \mathrm{R}$ e $\mathrm{D}$, sugerindo-se como valores iniciais $\mathrm{D}=0.3 \mathrm{e}$ $\mathrm{S}=20$, para o Tremolo, $\mathrm{S}=10$ para o Phaser e $\mathrm{D}=0.9$ e $\mathrm{R}=10$ para o Auto - Wah.

\section{Conclusões}

Foram apresentados exemplos educativos de utilização dos recursos de geração de som disponíveis no software Mathematica e sua aplicação no ensino de Física, com possível extensão ao da Música. Para se trabalhar o processo de aprendizado, foi utilizada uma única função do ambiente de programação simbólica, denominada Play, que pode ser prontamente modificada on-line. A demonstração de conceitos geralmente vistos apenas nos livros de Física, como o fenômeno de batimento, mostra como os recursos de áudio do Mathematica podem ser utilizados em um nível de conhecimento mais básico. A metodologia desenvolvida permite que atividades de aprendizado em nível mais avançado, como a modelagem e análise do Tremolo, Phaser e Auto-wah, possam ser autonomamente trabalhadas pelo aluno [7], permitindo-lhe modelar e interpretar efeitos acústicos ainda mais complexos, estimulando assim sua criatividade. O professor poderá utilizar este ambiente e complementar o processo de aprendizado discutindo na classe aspectos artístico-culturais, podendo implementar algoritmos simbólicos de caráter mais abrangente e pedagógico.

\section{Referências}

[1] Johnston, I. (1989). Measured Tones. IOP Publishing.

[2] Helmholtz, H. (1954). On the Sensations of Tone. Dover Publications, Inc.

[3] Ramalho, G. L., Ganascia, J.G (1994). Simulating Creativity in Jazz Performance. In: The Twelfth National

\footnotetext{
${ }^{1}$ As letras que indicam cada parâmetro vêm dos nomes utilizados pelos fabricantes de pedais e racks de efeitos. D vêm de $D$ epth, S de Speed e R de Rate. Eles são geralmente controlados através de potenciômetros, com unidades arbitrárias que variam de acordo com fabricantes e modelos.
} 
Conference on Artificial Intelligence (AAAI 94), Seattle. p. 108-113.

[4] Cruz, M. A. S., Manzolli, J., Damiani, F. (2001). Espectro Sonoro Explorado com Sistemas de Funções Iterativas. In: VIII Brazilian Symposium on Computer Music (SBC\&M 2001), Fortaleza.

[5] Halliday, D., Resnick, R., Walker, J. (1993). Fundamentals of Physics, 4th Edition, Extended Version.
John Wiley \& Sons, Inc.

[6] Gomes, A., Neves, A. (1993). Tecnologia Aplicada à Música. Érica.

[7] Ramos, E. M. F. (1995). Análise ergonômica do sistema hiperNet buscando o aprendizado da cooperação $e$ da autonomia. In: I Congresso de Informática Educativa do Mercosul. 